\title{
WATER QUALITY RETRIEVAL AND ALGAL BLOOM DETECTION USING HIGH- RESOLUTION CUBESAT IMAGERY
}

\author{
Milad Niroumand-Jadidi ${ }^{1, *}$, Francesca Bovolo ${ }^{1}$ \\ ${ }^{1}$ Digital Society Center, Fondazione Bruno Kessler, Via Sommarive, 18 I-38123, Trento, Italy; (mniroumand, bovolo)@ fbk.eu
}

Commission III, WG III/9

KEY WORDS: Water Quality, CubeSat Imagery, PlanetScope, Algal Bloom, Chlorophyll-a, Suspended Sediment, Lake

\begin{abstract}
:
Recent advancements in developing small satellites known as CubeSats provide an increasingly viable means of characterizing the dynamics of inland and nearshore waters with an unprecedented combination of high revisits ( $<1$ day) with a high spatial resolution (meter-scale). Estimation of water quality parameters can benefit from the very high spatiotemporal resolution of CubeSat imagery for monitoring subtle variations and identification of hazardous events like algal blooms. In this study, we present the first study on retrieving lake chlorophyll-a (Chl-a) concentration and detecting algal blooms using imagery acquired by the PlanetScope constellation which is currently the most prominent source of CubeSat data. Moreover, the concentration of total suspended matter (TSM) is retrieved that is an indicator of turbidity. The retrievals are based upon inverting the radiative transfer model. The low spectral resolution (four bands) of PlanetScope imagery poses challenges for such a physics-based inversion due to spectral ambiguities in optically-complex waters like inland waters. To deal with this issue, the number of variable parameters is minimized through inverse modeling. Given the significance of having high-quality water-leaving reflectance for physics-based models, a variable parameter $\left(g_{d d}\right)$ is considered to compensate for the atmospheric and sun-glint artifacts. The results compared to the in-situ data indicate high potentials of PlanetScope imagery in retrieving water quality parameters and detection of algal blooms in our case study (Lake Trasimeno, Italy).
\end{abstract}

\section{INTRODUCTION}

A new era of high spatial resolution imaging has just emerged with the launch of CubeSat satellites. Owing to their small size and affordable launch costs, a constellation of hundreds of CubeSats has been made possible. Such a large number of CubeSat imagers overcomes the traditional trade-off between high spatial and high temporal resolutions (Cooley et al., 2017; Niroumand-Jadidi et al., 2020c). For instance, the recent PlanetScope constellation of satellites $(180+$ CubeSats) has paved the way for monitoring Earth resources including inland waters with a high spatial resolution $(3 \mathrm{~m})$ and on a daily basis (Planet, 2020). This unprecedented spatiotemporal resolution opens up new horizons for near real-time monitoring of biophysical processes in inland waters. This is of particular importance when the spatial resolution of common multispectral satellites (e.g., Sentinel-2 and Landsat-8) is too coarse for studying small bodies of water like rivers and small lakes. The appeal of daily acquisitions is the potential to provide temporally continuous information on fast-evolving biophysical parameters like water quality indicators. Moreover, processes like eutrophication can be monitored in a timely manner, which facilitates the detection of harmful algal blooms. Although high spatial and temporal resolutions of the PlanetScope constellation are very attractive for studying inland waters, the low spectral resolution (four bands) poses challenges for the estimation of biophysical parameters (Niroumand-Jadidi et al., 2020c). This is because of the spectral ambiguity resultant from the combination of various parameters that define the shape and magnitude of water-leaving spectra (Defoin-Platel and Chami, 2007). Some of these parameters are concerned with the quality of water such as chlorophyll-a (Chl-a), total suspended matter (TSM), and colored dissolved organic matter (CDOM). In optically-shallow waters that light can penetrate the entire water column, there are also influences from the water depth (bathymetry) as well as the substrate (Niroumand-Jadidi et al., 2020a). Moreover, there are

\footnotetext{
* Corresponding author
}

concerns about the radiometric quality and stability of PlanetScope imagery for aquatic applications (Cooley et al., 2017). The radiometric quality of optical imagery is of particular importance in retrieving biophysical parameters (e.g., in-water constituents) due to the low signal-to-noise ratio SNR $(<15 \%)$ over water bodies (Moses et al., 2012; Niroumand-Jadidi et al., 2018). The low SNR is related to the high absorption of signal by pure water and thus pronounced effect of the noise introduced by atmospheric effects.

There are still sparse studies on the applications of PlanetScope imagery in retrieving biophysical parameters in inland waters. This is mainly due to the mentioned limitations of PlanetScope imagery in terms of spectral and radiometric resolutions. Moreover, the capacity of the constellation is maximized only recently by launching more CubeSat imagers. Thus, there is a need to examine the potentials of this new source of data in retrieving parameters like water quality indicators given all the aforementioned limitations. The existing few studies are mostly focused on the estimation of bathymetry based on regression models (Gabr et al., 2020; Poursanidis et al., 2019). A semiempirical approach is applied for the estimation of water turbidity in coastal areas (Vanhellemont, 2019). The empirical (regression-based) methods require site-specific in-situ data for training the model (Niroumand-Jadidi et al., 2019; NiroumandJadidi and Vitti, 2016). On the other hand, there are physicsbased methods that retrieve the biophysical parameters by inverting the radiative transfer model (Gege, 2014). The application of these methods does not require in-situ measurements. However, knowledge about inherent optical properties (IOPs) of the water column and bottom albedo (in the case of optically-shallow waters) is needed to account for absorption and scattering properties through physics-based modeling. The only existing study based on a physics-based inversion of PlanetScope imagery is a work conducted in the Venice lagoon for analyzing the impact of COVID-19 lockdown as well as an extreme flood on the water quality (Niroumand- 
Jadidi et al., 2020c). That study demonstrated the potentials of PlanetScope imagery in retrieving TSM concentration and bathymetry in the lagoon. In this study, we perform the first analyses on the utility of 4-band PlanetScope imagery in retrieving water quality parameters including TSM and Chl-a as well as detection of algal bloom based on a physics-based approach. Toward this end, we adapt the Water Color Simulator (WASI) processor (Gege, 2014) for inverting PlanetScope imagery in a turbid lake (Lake Trasimeno, Italy). Chl-a is an indicator of phytoplankton biomass that allows for the detection of harmful blooms. TSM is associated with organic and mineral suspended solids and is closely associated with water turbidity and Secchi depth (Soomets et al., 2020). Accurate information on spatiotemporal dynamics of Chl-a and TSM can contribute to studies of aquatic habitats, aquaculture, biogeochemical cycles as well as management of drinking and agricultural water supply and recreation activities (Maeda et al., 2019; Soomets et al., 2020).

The rest of the manuscript is organized as follows: the case study and dataset are introduced in Section 2. Section 3 describes the physics-based inversion method and its parametrization. Experimental results and discussions are provided in Section 4. Finally, conclusions and recommendations for future works are presented in Section 5.

\section{CASE STUDY AND DATASET}

Lake Trasimeno is selected as our case study, which is a turbid (Secchi depth $\sim 1.1 \mathrm{~m}$ ) and eutrophic lake in Central Italy (Bresciani et al., 2020). The southeast corner of the lake is populated with aquatic vegetation, which is excluded from our analyses (Figure 1). The ecosystem of Lake Trasimeno is threatened by seasonal algal blooms that occur mostly from July to September (Bresciani et al., 2020; Giardino et al., 2015). There are two stations in the lake operated by the local environmental agency providing Chl-a measurements every two months. Chl-a concentration varies in the range of $2-40 \mathrm{mg} / \mathrm{m}^{3}$ according to long-term records at these stations. This high variation of Chl-a pronounces the advantage of CubeSat imagers in providing daily acquisitions that can contribute to the timely management of inland waters like Lake Trasimeno. The mean concentration of TSM is about $10 \mathrm{~g} / \mathrm{m}^{3}$ (Bresciani et al., 2020) and $a_{C D O M}(440)$ can be considered as $0.3 \mathrm{~m}^{-1}$ in average (Giardino et al., 2015).

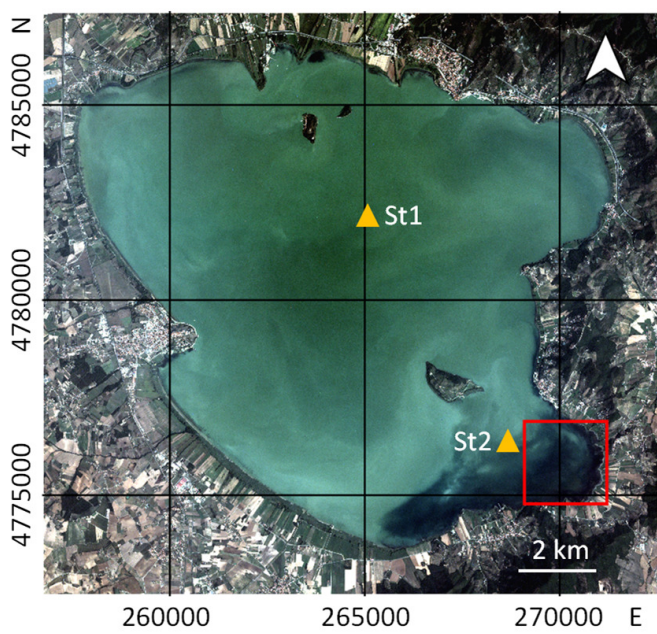

Figure 1. PlanetScope image of Lake Trasimeno acquired on 2 September 2019. The red box shows the open bay affected by the aquatic vegetation. In-situ stations (St1 and St2) are shown by yellow symbols.
We analyze mosaics from the PlanetScope constellation dated 2 September 2019 and 25 May 2020 made of three and two tiles, respectively. The images are acquired with a ground sampling size of $3.7 \mathrm{~m}$ and orthorectified to $3 \mathrm{~m}$ spatial resolution. The PlanetScope data provide four spectral bands in the visible (RGB) and near-infrared (NIR) portions of the spectrum with a 12-bit radiometric resolution (Table 1). Here, we process the level-3B products, which are the surface reflectance images. The $6 \mathrm{~S}$ radiative transfer model is used by the provider to perform the atmospheric correction (Planet, 2020).

\begin{tabular}{|l|l|}
\hline Bands & Spectral range \\
\hline Blue & $455-515 \mathrm{~nm}$ \\
Green & $500-590 \mathrm{~nm}$ \\
Red & $590-670 \mathrm{~nm}$ \\
NIR & $780-860 \mathrm{~nm}$ \\
\hline
\end{tabular}

Table 1. Spectral bands of PlanetScope imagery.

The in-situ measurements are available for image acquisition dates from the stations. In this context, the average Chl-a concentration in the stations is recorded as $\sim 36 \mathrm{mg} / \mathrm{m}^{3}$ and 3.4 $\mathrm{mg} / \mathrm{m}^{3}$ for 2 September 2019 and 25 May 2020, respectively. The extremely high concentration of Chl-a (on the order of 10 times) for 2 September 2019 is an indication of an algal bloom. Thus, the selected images provide the possibility of examining the potentials of PlanetScope imagery in mapping a wide range of Chl-a concentrations and detecting algal blooms.

\section{METHOD}

We adapt the physics-based WASI processor (Gege, 2014) for inversion of the PlanetScope imagery to derive the in-water constituents. WASI is a flexible model that can be adapted to different bio-optical conditions either in optically-shallow, i.e., considerable bottom-reflected radiance, or optically-deep waters, i.e., negligible bottom-reflected radiance (Gege, 2014; Niroumand-Jadidi et al., 2020b). WASI simulates a set of waterleaving spectra by varying different parameters (e.g., the concentration of constituents). The simulated spectra are compared with a given observed (image) spectrum to find the best match. The values of variable (fit) parameters yielding to the optimal spectrum match are considered as retrieved values. This process runs individually over the entire pixels of the water body. In the next subsections, we explain the main physical models of WASI for radiative transfer simulations, the cost function for performing the spectrum matching, and the adaptation to process PlanetScope imagery. Moreover, the parametrization and initialization of fit parameters are provided for our case study.

\subsection{Inverse Modeling with WASI}

The reflectance $R_{r s}(\lambda)$ recorded by optical imagery can be considered as the contribution from (i) remote sensing reflectance $r_{r s}$, which is the ratio of upwelling radiance to downwelling irradiance just below the water surface (Gege, 2004; Mobley, 1994), and (ii) the reflection from the water surface $R_{r s}^{\text {surf }}(\lambda)$ :

$$
R_{r s}(\lambda)=\frac{\zeta \cdot r_{r s}(\lambda)}{1-\Gamma \cdot r_{r s}(\lambda)}+R_{r s}^{\text {surf }}
$$

where $\zeta \approx 0.52=$ water-to-air radiance divergence

$$
\Gamma \approx 1.6=\text { reflection of upwelling radiance at }
$$
water surface

For the majority of pixels, there is no bottom effect on the waterleaving spectra due to the high level of turbidity in our case study. 
Thus, simulation of $r_{r s}(\lambda)$ is performed based on an opticallydeep water model (Albert, 2004; Albert and Mobley, 2003):

$r_{r s}^{\text {deep }}(\lambda)=0.0512 \cdot u(\lambda) \cdot(1+4.6659$.

$\left.u(\lambda)-7.8387 \cdot u(\lambda)^{2}+5.4571 \cdot u(\lambda)^{3}\right) \cdot\left(1+\frac{0.1098}{\cos \theta_{\text {sun }}^{\prime}}\right)$.

$\left(1+\frac{0.4021}{\cos \theta_{v}^{\prime}}\right) \cdot\left(1-0.0044 v_{w}\right)$.

where $\quad \theta_{\text {sun }}^{\prime}=$ solar zenith angle in the water

$\theta_{v}^{\prime}=$ viewing zenith angle in the water

$v_{w}=$ wind speed

We assume $v_{w}=0 \mathrm{~ms}^{-1}$ as the wind speed has minimal impact on $r_{r s}^{\text {deep }}(\lambda)$. The function $u(\lambda)$ characterizes the in-water absorption $a(\lambda)$ and backscattering properties $b_{b}(\lambda)$ :

$$
u(\lambda)=\frac{b_{b}(\lambda)}{a(\lambda)+b_{b}(\lambda)}
$$

Both $a(\lambda)$ and $b_{b}(\lambda)$ can be expressed in terms of contributions of pure water and different optically-active constituents:

$$
\begin{array}{rr}
a(\lambda)=a_{w}(\lambda)+a_{C D O M}\left(\lambda_{0}\right) \cdot \exp \left\{-S_{C D O M} \cdot\right. & (4) \\
\left.\left(\lambda-\lambda_{0}\right)\right\}+C_{p h y} \cdot a_{p h y}^{*}(\lambda)+C_{N A P} \cdot a_{N A P}^{*}\left(\lambda_{0}\right) \cdot \exp \left\{-S_{N A P}\right. & \left.\left(\lambda-\lambda_{0}\right)\right\}
\end{array}
$$

$$
b_{b}(\lambda)=b_{b, w}(\lambda)+C_{p h y} \cdot b_{b, p h y}^{*}(\lambda)+C_{N A P} \cdot b_{b, N A,}^{*}
$$

where $\quad a_{w}(\lambda)=$ absorption coefficients of pure water $b_{b, w}(\lambda)=$ backscattering coefficients of pure water $C_{p h y}=$ phytoplankton (Chl-a) concentration

$C_{N A P}=$ non-algal particlues (NAP) or TSM concentration

$a_{C D O M}\left(\lambda_{0}\right)=$ absorption coefficient of CDOM at the wavelength $\lambda_{0}=440 \mathrm{~nm}$

$S_{N A P}=$ spectral slope of CDOM

$a_{p h y}^{*}(\lambda)=$ specific absorption spectrum of phytoplankton

$a_{N A P}^{*}\left(\lambda_{0}\right)=$ specific absorption coefficient of NAP at $\lambda_{0}=440 \mathrm{~nm}$

$S_{C D O M}=$ spectral slope of CDOM

$b_{b, N A P}^{*}(\lambda)=$ specific backscattering coefficient of NAP

Then, a three-component model for the sky radiance reflected in the viewing direction is employed to simulate the reflections at the water surface (Gege, 2014; Gege and Groetsch, 2016):

$$
\begin{aligned}
& R_{r s}^{\text {surf }}(\lambda)=\rho_{L}\left(\theta_{v}\right) \times \frac{L_{s k y}(\lambda)}{E_{d d}(\lambda)+E_{d s r}(\lambda)+E_{d s a}(\lambda)}, \\
& L_{s k y}(\lambda)=g_{d d} E_{d d}(\lambda)+g_{d s r} E_{d s r}(\lambda)+g_{d s a} E_{d s a}(\lambda)
\end{aligned}
$$

where $\rho_{L}\left(\theta_{v}\right)=$ Fresnel reflection for a viewing angle of $\theta_{v}$ $E_{d d}(\lambda)=$ direct downwelling irradiance from sun disk $E_{d s r}(\lambda)=$ diffuse downwelling irradiance of Rayleigh scattering

$E_{d s a}(\lambda)=$ diffuse downwelling irradiance of aerosol scattering

$g_{d s r}=1 / \pi=$ sky radiance fractions due to molecule $g_{d s a}=1 / \pi=$ sky radiance fraction by aerosol scattering $g_{d d}=$ sky radiance fraction due to direct solar radiation
We consider a least-squares cost function $(\Delta)$ to assess the agreement between simulated and observed (image) spectra. Thus, the values of fit parameters minimizing the cost function are the inversion retrievals:

$$
\Delta=\frac{1}{N} \sum_{i=1}^{N}\left|R_{r s}^{\text {measured }}\left(\lambda_{i}\right)-R_{r s}^{\text {simulated }}\left(\lambda_{i}\right)\right|^{2}
$$

where

$$
\begin{aligned}
& R_{r s}^{\text {simulated }}(\lambda)=\text { simulated spectrum } \\
& R_{r s}^{\text {measured }}(\lambda)=\text { measured spectrum } \\
& \lambda_{i}=\text { central wavelength of band number } i
\end{aligned}
$$

\subsection{Parametrization and Initialization of WASI}

Different combinations of fit parameters values may lead to similar spectra not distinguishable by the low spectral resolution of PlanetScope imagery (four bands). Varying more parameters makes this problem more critical. Thus, it is important to minimize the number of fit parameters to mitigate the problem of spectral ambiguity through the inversion (Niroumand-Jadidi et al., 2020c). Therefore, the parameters that their variations are small and thus have less impact on the water-leaving spectra should be treated as fixed. In this context, the concentration of Chl-a $\left(C_{p h y}\right)$, concentration of TSM $\left(C_{N A P}\right)$, absorption of CDOM $a_{C D O M}(440)$, and $g_{d d}$ are considered as fit parameters. The $g_{d d}$ is treated as a variable parameter to mitigate the sunglint and atmospheric artificats. The $g_{d d}$ correction is essential for processing PlanetScope surface reflectance products given that the atmospheric correction is not developed for the aquatic applications and associated SNR poblems. The initial values of the fit parameters are based on pre-fit analyses and the knowledge about the range of parameters in the case study (Table 2). Note that two initial values are considered for $C_{p h y}$ as the initialization with normal condition value $\left(5 \mathrm{mg} / \mathrm{m}^{3}\right)$ did not provide small $\Delta$ through the spectrum matching for the algal bloom condition $(2$ September 2019). The pure water properties of $a_{w}(\lambda)$ and $b_{b, w}(\lambda)$ are taken from (Kou et al., 1993; Pope and Fry, 1997), and (Morel, 1974), respectively. The fixed IOP parameters (Table 2) are based on the in-situ measurements in Lake Trasimeno (Giardino et al., 2015). The wavelength-dependent $a_{\text {phy }}^{*}(\lambda)$ is also specific to Lake Trasimeno which is shown in Figure 2 (Giardino et al., 2015).

\begin{tabular}{|l|l|}
\hline \multicolumn{1}{|c|}{ Fixed parameters } & \multicolumn{1}{c|}{$\begin{array}{c}\text { Fit parameters and } \\
\text { (initial values) }\end{array}$} \\
\hline$S_{N A P}=0.011 \mathrm{~nm}^{-1}$ & $C_{p h y}\left(5,25 \mathrm{mg} / \mathrm{m}^{3}\right)$ \\
$a_{N A P}^{*}(440)=0.0248 \mathrm{~m}^{2} \mathrm{~g}^{-1}$ & $C_{N A P}\left(7 \mathrm{~g} / \mathrm{m}^{3}\right)$ \\
$S_{C D O M}=0.016 \mathrm{~nm}^{-1}$ & $a_{C D O M}(440)\left(0.2 \mathrm{~m}^{-1}\right)$ \\
$b_{b, N A P}^{*}(\lambda)=0.01271 \mathrm{~m}^{2} \mathrm{~g}^{-1}$ & $g_{d d}\left(0.6 \mathrm{sr}^{-1}\right)$ \\
\hline
\end{tabular}

Table 2. Fixed and fit parameters considered for WASI-based inversion of PlanetScope imagery in Lake Trasimeno.

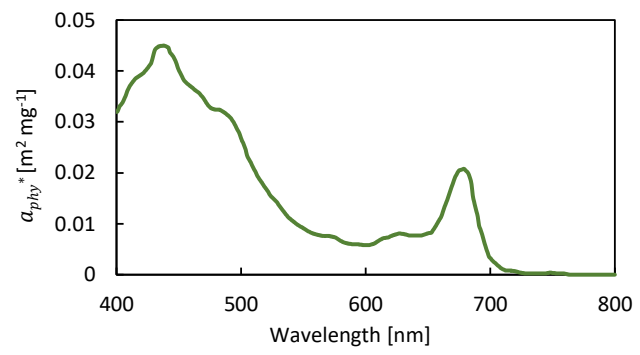

Figure 2. The specific absorption spectrum of phytoplankton $a_{p h y}^{*}(\lambda)$ measured in Lake Trasimeno. 


\section{RESULTS AND DISCUSSION}

The Chl-a maps derived with the WASI adaptation to PlanetScope imagery are shown in Figure 3. The open bay colonized by the aquatic vegetation is masked out as the deepwater inversion is not appropriate for this region having influences from the bottom on the water-leaving spectra. The concentration of Chl-a is extremely high for the image acquired in September 2019. The average of retrieved Chl-a at the stations ( $5 \times 5$ pixels) for this date is $\sim 26 \mathrm{mg} / \mathrm{m}^{3}$ whereas it is on the order of $\sim 3.3 \mathrm{mg} / \mathrm{m}^{3}$ for the image acquired in May 2020 . This is in line with the available information from the in-situ measurements that indicate an algal bloom acquiring late summer 2019 (Section 2).
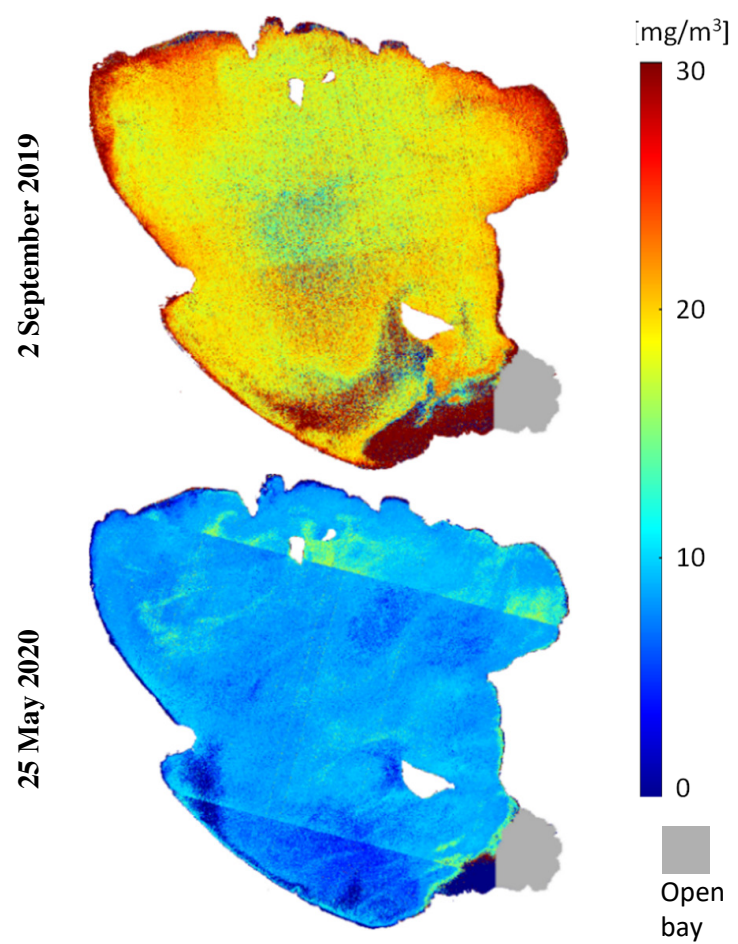

Figure 3. Chl-a maps of Lake Trasimeno derived from PlanetScope imagery.

Figure 4 shows retrieved-to-observed matchups that indicate the high potential of PlanetScope imagery in mapping a wide range of Chl-a concentrations.

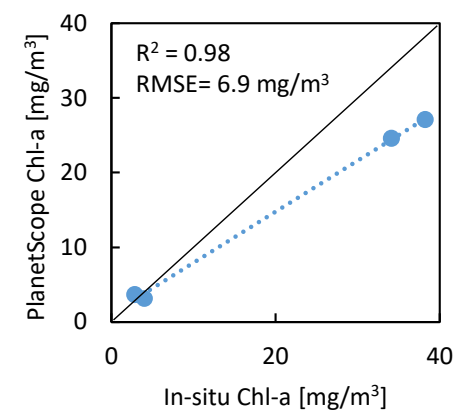

Figure 4. Matchup validation of the estimated Chl-a from PlanetScope imagery.

Figure 5 illustrates the TSM maps of Lake Trasimeno derived from PlanetScope imagery with the proposed WASI adaptation. The estimated values are in agreement with the available information about the average TSM concentration reported in the literature $\left(\sim 10 \mathrm{~g} / \mathrm{m}^{3}\right.$, see Section 2$)$. The comparison of two TSM maps also shows that the pattern and the level of TSM can have large changes over time. The spatiotemporal average $g_{d d}$ for two acquisition dates is retrieved on the order of $0.94 \mathrm{sr}^{-1}$ through the inversion. The $g_{d d}$ is typically in the range of $0-0.03 \mathrm{sr}^{-1}$ (Gege and Groetsch, 2016). Thus, the high value of $g_{d d}$ indicates sizable atmospheric and sun-glint artifacts on the surface reflectance products of PlanetScope imagery. This finding suggests that the path radiance is underestimated through the atmospheric correction that the WASI-base inversion mitigates this effect. This is in line with the previous study on the inversion of PlanetScope imagery (Niroumand-Jadidi et al., 2020c).

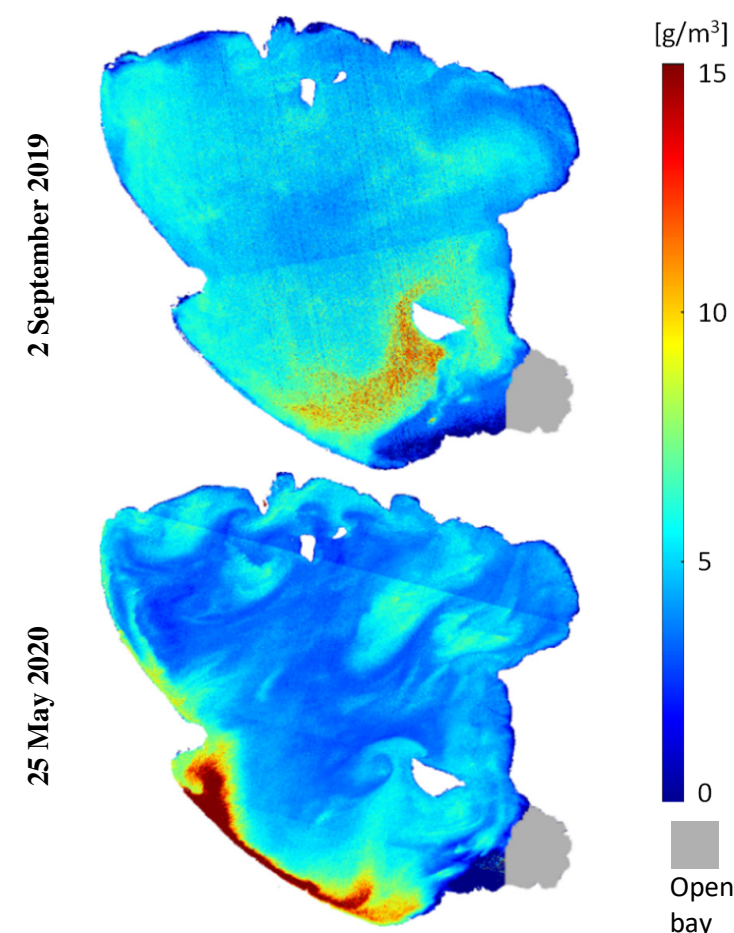

Figure 5. TSM maps of Lake Trasimeno derived from PlanetScope imagery.

\section{CONCLUSIONS AND OUTLOOKS}

The CubeSat satellites and particularly the recent PlanetScope constellation offer new opportunities for monitoring inland and nearshore coastal waters. This optimism is linked to the high spatiotemporal resolution of the PlanetScope data (i.e., daily revisits at a $3 \mathrm{~m}$ spatial resolution). This is very favorable for monitoring in-water constituents which are very dynamic in inland waters. However, the low spectral resolution and the issues related to the radiometric quality and atmospheric correction are key challenges for the application of these images in aquatic systems. This study performed the first analyses on the utility of 4-band PlanetScope imagery in retrieving lake water quality parameters and detecting algal blooms based upon a physics-based approach. The adapted WASI model provided promising results of Chl-a and TSM concentrations by incorporating the parameter $g_{d d}$ for mitigating the atmospheric and sun-glint artifacts. The estimates of the constituents are in good agreement with the in-situ observations. More specifically, the potential of PlanetScope imagery in detecting and mapping the algal blooms is demonstrated that is of particular importance in the management of lakes and associated ecosystem services. Although the artifacts of the standard surface reflectance products are minimized in this study by $g_{d d}$ parameter, dedicated 
studies are required to develop more accurate atmospheric correction for aquatic applications. More studies are also needed to examine the constituent retrieval from PlanetScope imagery in different water types (e.g., oligotrophic, CDOM-rich, etc.) and considering more in-situ data for matchup analysis. Although we employed PlanetScope imagery in this study, the methodology can be applied to any high-resolution imagery with some adaptations. The imagery can be either from traditional highresolution satellites (e.g., IKONOS and GeoEye) or other CubeSat constellations. In particular, there are upcoming constellations of high-resolution satellites like WorldView legion planned to be launched in 2021 by the MAXAR company that can further enhance the monitoring of rapidly changing water quality conditions in inland waters.

\section{ACKNOWLEDGEMENTS}

The authors appreciate the Education and Research Program of Planet Inc. that provided us the PlanetScope imagery. We also acknowledge in-situ Chl-a data provided by the regional environmental protection agency of Umbria (ARPA Umbria).

\section{REFERENCES}

Albert, A., 2004. Inversion technique for optical remote sensing in shallow water. University of Hamburg, Germany.

Albert, A., Mobley, C., 2003. An analytical model for subsurface irradiance and remote sensing reflectance in deep and shallow case-2 waters. Opt. Express 11, 2873.

Bresciani, M., Pinardi, M., Free, G., Luciani, G., Ghebrehiwot, S., Laanen, M., Peters, S., Della Bella, V., Padula, R., Giardino, C., 2020. The Use of Multisource Optical Sensors to Study Phytoplankton Spatio-Temporal Variation in a Shallow Turbid Lake. Water 12, 284.

Cooley, S., Smith, L., Stepan, L., Mascaro, J., 2017. Tracking Dynamic Northern Surface Water Changes with High-Frequency Planet CubeSat Imagery. Remote Sens. 9, 1306.

Defoin-Platel, M., Chami, M., 2007. How ambiguous is the inverse problem of ocean color in coastal waters? J. Geophys. Res. 112, C03004.

Gabr, B., Ahmed, M., Marmoush, Y., 2020. PlanetScope and Landsat 8 Imageries for Bathymetry Mapping. J. Mar. Sci. Eng. 8,143 .

Gege, P., 2004. The water color simulator WASI: An integrating software tool for analysis and simulation of optical in situ spectra. Comput. Geosci. 30, 523-532.

Gege, P., 2014. WASI-2D: A software tool for regionally optimized analysis of imaging spectrometer data from deep and shallow waters. Comput. Geosci. 62, 208-215.

Gege, P., Groetsch, P., 2016. A spectral model for correcting sun glint and sky glint. In: Proc. Ocean Optics XXIII. 23-28. October. 2016, Victoria, Canada.

Giardino, C., Bresciani, M., Valentini, E., Gasperini, L., Bolpagni, R., Brando, V.E., 2015. Airborne hyperspectral data to assess suspended particulate matter and aquatic vegetation in a shallow and turbid lake. Remote Sens. Environ. 157, 48-57.

Kou, L., Labrie, D., Chylek, P., 1993. Refractive indices of water and ice in the 065- to 25- $\mu \mathrm{m}$ spectral range. Appl. Opt. 32, 3531.
Maeda, E.E., Lisboa, F., Kaikkonen, L., Kallio, K., Koponen, S., Brotas, V., Kuikka, S., 2019. Temporal patterns of phytoplankton phenology across high latitude lakes unveiled by long-term time series of satellite data. Remote Sens. Environ. 221, 609-620. Mobley, C.D., 1994. Light and water: radiative transfer in natural waters. Academic Press.

Morel, A., 1974. Optical Properties of Pure Water and Pure sea Water. Opt. Asp. Oceanogr. 1.

Moses, W.J., Bowles, J.H., Lucke, R.L., Corson, M.R., 2012. Impact of signal-to-noise ratio in a hyperspectral sensor on the accuracy of biophysical parameter estimation in case II waters. Opt. Express 20, 4309.

Niroumand-Jadidi, M., Bovolo, F., Bruzzone, L., 2019. Novel Spectra-Derived Features for Empirical Retrieval of Water Quality Parameters: Demonstrations for OLI, MSI, and OLCI Sensors. IEEE Trans. Geosci. Remote Sens. 57, 10285-10300.

Niroumand-Jadidi, M., Bovolo, F., Bruzzone, L., 2020a. SMART-SDB: Sample-specific multiple band ratio technique for satellite-derived bathymetry. Remote Sens. Environ. 251, 112091.

Niroumand-Jadidi, M., Bovolo, F., Bruzzone, L., 2020b. Water Quality Retrieval from PRISMA Hyperspectral Images: First Experience in a Turbid Lake and Comparison with Sentinel-2. Remote Sens. 12, 3984.

Niroumand-Jadidi, M., Bovolo, F., Bruzzone, L., Gege, P., 2020c. Physics-based Bathymetry and Water Quality Retrieval Using PlanetScope Imagery: Impacts of 2020 COVID-19 Lockdown and 2019 Extreme Flood in the Venice Lagoon. Remote Sens. 2020, Vol. 12, Page 2381 12, 2381.

Niroumand-Jadidi, M., Vitti, A., 2016. Optimal band ratio analysis of WorldView-3 imagery for bathymetry of shallow rivers (case study: Sarca River, Italy). In: International Archives of the Photogrammetry, Remote Sensing and Spatial Information Sciences - ISPRS Archives.

Niroumand-Jadidi, M., Vitti, A., Lyzenga, D.R., 2018. Multiple Optimal Depth Predictors Analysis (MODPA) for river bathymetry: Findings from spectroradiometry, simulations, and satellite imagery. Remote Sens. Environ. 218, 132-147.

Planet, 2020. Planet Imagery Product Specifications. https://assets.planet.com/docs/Planet_Combined_Imagery_Prod uct_Specs_letter_screen.pdf (accessed 7.10.20).

Pope, R.M., Fry, E.S., 1997. Absorption spectrum (380-700 nm) of pure water II Integrating cavity measurements. Appl. Opt. 36, 8710.

Poursanidis, D., Traganos, D., Chrysoulakis, N., Reinartz, P., 2019. Cubesats Allow High Spatiotemporal Estimates of Satellite-Derived Bathymetry. Remote Sens. 11, 1299.

Soomets, T., Uudeberg, K., Jakovels, D., Brauns, A., Zagars, M., Kutser, T., 2020. Validation and comparison of water quality products in baltic lakes using sentinel-2 msi and sentinel-3 OLCI data. Sensors 20.

Vanhellemont, Q., 2019. Daily metre-scale mapping of water turbidity using CubeSat imagery. Opt. Express 27, A1372. 